\title{
Effects of Exercise Training on Vascular Inflammatory Markers in High-fat Diet-induced Obese Rats
}

\author{
Kijin Kim* \\ Department of Physical Education, College of Physical Education, Keimyung University, Korea
}

\begin{abstract}
Received: January 23, 2020
Accepted: February 21, 2020

Published online: April 30, 2020

Keywords:

Exercise Training

Nitric Oxide

Obesity

Vascular Inflammation

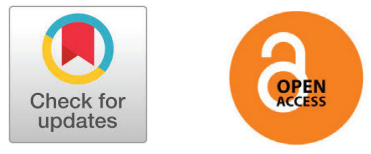

\section{ABSTRACT}

OBJECTIVES The purpose of this study was to confirm the positive effects on the inflammatory expression and functional changes of blood vessels by aerobic exercise training and dietary control in high-fat diet induced obese rats.

METHODS Forty 10-week-old male Wistar rats were treated with high-fat diet for 6 weeks, followed by 4 groups as sedentary + chow diet group (Chow), sedentary + high fat diet group (HFD), exercise + chow diet group (Chow-Ex), high fat diet + Exercise group (HFD-Ex) after obesity induction. After treadmill running exercise and dietary treatment were performed for 8 weeks, body composition, endurance performance and muscular endurance, blood glucose, CRP, NO, insulin, eNOS, iNOS, protein expression of IL- 6 and TNF- $a$ in aortic vascular tissues was analyzed.

RESULTS High-fat diet intake leads to an increase in body weight and visceral fat, an increase in insulin resistance and an increase in inflammation factors. The blood NO level was significantly higher $(p<0.05)$ in the HFD and Chow groups than in the non-exercise group. Blood insulin levels were significantly higher $(p<0.05)$ in the HFD group without exercise than in all other groups. Blood eNOS activity was significantly higher $(p<0.05)$ in the HFD and Chow groups than in the non-exercise group. The expression level of IL-6 protein in the vascular tissues was significantly higher $(p<0.05)$ in the HFD group without exercise than the other groups. The expression level of TNF- $a$ was significantly lower $(p<0.05)$ in the HFD and Chow groups than in the non-exercise group.

CONCLUSIONS The results of this study confirmed that exercise training through aerobic exercise and dietary control may help to reduce inflammatory expression and improve vascular endothelial function in high-fat diet induced obese rats.
\end{abstract}

๑) The Asian Society of Kinesiology and the Korean Academy of Kinesiology

\section{Introduction}

Obesity is a representative risk factor for cardiovascular disease, which is an important cause of vascular inflammation and dysfunction. In particular, obesity is a direct factor related to increased body fat, inflammation profile, reactive oxygen species (ROS) production, and nitric oxide-mediated dysfunction of vascular endothelium [1,2]. Vascular

*Correspondence: Kijin Kim, Department of Physical Education, College of Physical Education, Keimyung University, 1095 Dalgubeuldero, Dalseo-gu, Daegu, Korea; Tel: +82-053-580-5256; Fax: +82-053-580-5314; E-mail: kjk744@kmu.ac.kr inflammation and dysfunction due to obesity have been widely reported to have a negative effect on increasing the incidence of cardiovascular diseases including hypertension, dyslipidemia, diabetes and cancer $[1,3]$. Therefore, in order to prevent cardiovascular diseases caused by vascular inflammation and dysfunction, analysis of the effects of obesity and related mechanisms and various methods for their treating have been widely attempted.

Mechanisms associated with vascular inflammation and decreased function due to obesity include low levels of inflammation caused by increased inflammation-induced 
cytokines secreted from adipose tissue around blood vessels, oxidative stress and decreased nitric oxide (NO) availability [4]. In particular, the inflammatory response considered to be the most representative factor related to vascular function control is the increased secretion of inflammatory cytokines from adipose tissue, which is a mediator of the clinical relationship between cardiovascular disease and diabetes. It is considered to be the most important factor in the pathogenesis of both type 1 and type 2 diabetes [5]. Various methods for suppressing inflammation, which are the most representative risk factors of vascular function, have been sought. As a cause, interest in adipose tissue around blood vessels has been emphasized [6]. In the process of inflammation, adipose tissue around the blood vessels is a key organ that secretes inflammatory cytokines and chemokines and causes hypertension, arteriosclerosis, hyperlipidemia and diabetes [7]. Therefore, the more effective obesity treatment process is required to reduce the body fat and perivascular adipose tissue as well as to suppress the inflammation process.

Exercise training, which is the most representative method for obesity treatment, is known to be the most effective method for preventing and alleviating vascular inflammation and deterioration as well as obesity treatment, especially for improving inflammation and vascular endothelial damage and vascular relaxation has been considered specific help [8]. Improving cardiovascular risk indicators associated with dyslipidemia, hypertension and diabetes has been considered to help mitigate risk factors associated with chronic cardiovascular disease and cancer [9]. However, recent studies have shown that there is no clear effect on the reduction of low-level inflammation by regular exercise and improvement of risk indicators of cardiovascular disease in the elderly [10], and Carlsson et al. [11] suggested no effective results could be identified. In addition, Pedrinolla et al. [12] pointed out that exercise training alone insufficiently prevents and alleviates the effects of vascular inflammation and deterioration in aging and obesity. Ongoing research is needed to establish the evidence related to the necessity. In other words, it is necessary to have a clearer analysis on whether a combination of dietary treatment and exercise can alleviate vascular inflammation and dysfunction.
In this study, 10-week-old male Wistar rats were treated with 6 weeks of high fat diet for obesity, followed by 8 weeks of aerobic exercise training and dietary control. The purpose of this study was to identify the positive effects on the inflammatory expression and functional changes of blood vessels by the combination of exercise training and dietary control in high-fat diet induced obese rats.

\section{Methods}

\section{Subjects}

Forty male 10-week-old Wistar rats (weight, 300-330 g) were subject to obesity after 6 weeks of high fat diet. Four groups after induction of obesity, sedentary + chow diet group (Chow, $\mathrm{n}=10)$, sedentary + high fat diet group (HFD, $\mathrm{n}=$ 10 ), exercise + chow diet group (Chow-Ex, $n=10)$, high fat diet + Exercise group (HFD-Ex, $n=10$ ), were carried out to determine the effects of exercise training and diet composition. High-fat diets were $30 \%$ carbohydrate, $50 \%$ fat, $20 \%$ protein, and $65 \%$ carbohydrate, $15 \%$ fat, and $20 \%$ protein. Two rats were kept in one cage $(20.7 \times 35 \times 17 \mathrm{~cm})$, and the temperature of the cage was maintained at $21^{\circ} \mathrm{C}$. The light phase and dark phase were adjusted to 12 hours each, and the dark phase was adjusted from 7:00 am to 7:00 pm. This animal protocol has approved the deliberation of the Animal Experimental Ethics Committee of Daegu Techno Park Bio Health Convergence Center (Approved number: BHCC-IACUC-2016-01).

\section{Experimental Procedures}

Exercise training program: The exercise protocol was partially modified by the method of Ivy et al. [13]. The treadmill run was performed three times a week with medium intensity using an electric laboratory treadmill (Quinton Instrument, Seattle, WA). To adapt the exercise program for the first week, perform 5 minutes at $10 \mathrm{~m} / \mathrm{min}$ speed and $0^{\circ}$ slope, then gradually increase the treadmill speed, slope and time, and from 2 weeks to $20 \mathrm{~m} / \mathrm{min}$ at $8^{\circ}$ slope. Run for 30 minutes to maintain exercise level until the end of the experiment. All treatments were conducted between 9:00 am and 10:00 am.

Endurance exercise test: After 4 weeks of treatment, the endurance exercise test was performed using Simi et al. [14]. 
The treadmill speed was set at $22 \mathrm{~m} / \mathrm{min}$ and the slope was set at $0 \%$ to measure the time from start to exhaustion when a person refuses to run or posture even after three or more encouragements.

Muscle endurance test: Suspension test was performed using the method of measuring muscle function suggested by Park et al. [15]. Muscle function was measured by measuring the time (sec) the test animal is suspended by installing a parallel bar ( $5 \mathrm{~mm}$ thick) of about $50 \mathrm{~cm}$ height from the ground. The average of the values obtained by performing three discontinuously was determined as muscular endurance.

Oral glucose tolerance test (OGTT): After 8 weeks of treatment, 48 hours of rest and 12 hours of fasting were excluded to rule out last-bout exercise effects. After resting blood through the tail, $50 \%$ glucose solution was orally administered at $1 \mathrm{~g} / 1 \mathrm{~kg}$. Then, at 15, 30 and 60 minutes, 300 $\mu \mathrm{l}$ of blood was collected from the tail. The collected blood was subjected to anticoagulant treatment ( $5 \mu$ heparin) and then centrifuged (1500 g, 15 minutes) to extract only plasma and stored at $-80^{\circ} \mathrm{C}$. until analysis. Rats with blood samples were injected subcutaneously with $0.9 \%$ saline solution $(2.5 \mathrm{~mL})$ to replenish the lost plasma.

Tissue extraction and blood collection: After a total of 8 weeks of treatment, fasting was performed for 12 hours, anesthetized with pentobarbital sodium ( $5 \mathrm{mg} / 100 \mathrm{~g}$ of body mass), and body composition was measured using DEXA for bovine animals. Thereafter, $5 \mathrm{~mL}$ of blood was collected from the abdominal artery of the abdominal cavity. After blood collection, visceral fat was removed from each site and weighed.

Blood variables analysis: Blood glucose levels were measured using an automatic blood glucose analyzer (YSI 2300 STAT plus, Springfield, USA) using whole blood. Blood collected from the abdominal cavity was separated from serum and enzymatic analysis using an ELISA kit (St. Louis, MO) of Sigma, nitric oxide (NO, KGE001, P193798), endothelial nitric oxide synthase (eNOS, E-EL-R0367, 657N6E1YPP) and inducible nitric oxide synthase (iNOS, E-EL-R0520, SKUF688U1T) levels were analyzed, respectively. Serum C-reactive protein (CRP) levels were measured by immunoassay using the N High Sensitivity CRP kit from the FDA (Dade Behring GmbH, Germany) and the Behring Nephelometer 100 analyzer (Messer Griesheim GmbH, Germany).

Western blotting: The method of Lowry et al. [16] was used for protein assay. The samples were dissolved in Laemmli buffer and electrophoresis was performed in SDSpolyacrylamide gel. In the immunoblotting using antibody, we measured interleukin-6 (IL-6) (Santa Cruz Bio, sc-1265), tumor necrosis factor- $\alpha$ (TNF- $\alpha$ ) (Santa Cruz Bio, sc-1350) and GAPDH (Santa Cruz, sc-47778), which were visualized using the ECL after an antibody treatment and being quantified by densitometry (sigma-plot 8.0 system).

\section{Statistical Analysis}

All data were calculated using mean and standard deviation (Mean \pm SD) for each group using SigmaPlot 12.0 statistical analysis program. One-way ANOVA was performed to analyze the differences among the groups, and two-way repeated ANOVA was performed to analyze the difference of OGTT results between treatment and time. The post hoc test was performed using Tukey's method and the statistical significance level was $\alpha=.05$.

\section{Results}

As the measurement results of body composition are shown in Fig. 1, the HFD group without exercise was significantly $(\mathrm{p}<0.05)$ highest in both body weight and visceral fat mass. Body weight was significantly higher $(\mathrm{p}<0.05)$ in the HFD group than in the Chow group in the non-exercise group. In the exercise group, the HFD group was higher than the Chow group, but there was no significant difference. Visceral fat content was also significantly higher $(\mathrm{p}<0.05)$ in the HFD group than in the Chow group in the non-exercise group, and the HFD group was higher than the Chow group in the exercise group, but there was no significant difference.

The results of physical fitness are shown in Fig. 2, the duration of exercise to all-out was significantly higher ( $p$ $<0.05)$ in both Chow-Ex and HFD-Ex groups than the HFD and Chow groups who did not exercise. At the suspension time, both the HFD and Chow groups who exercised were 

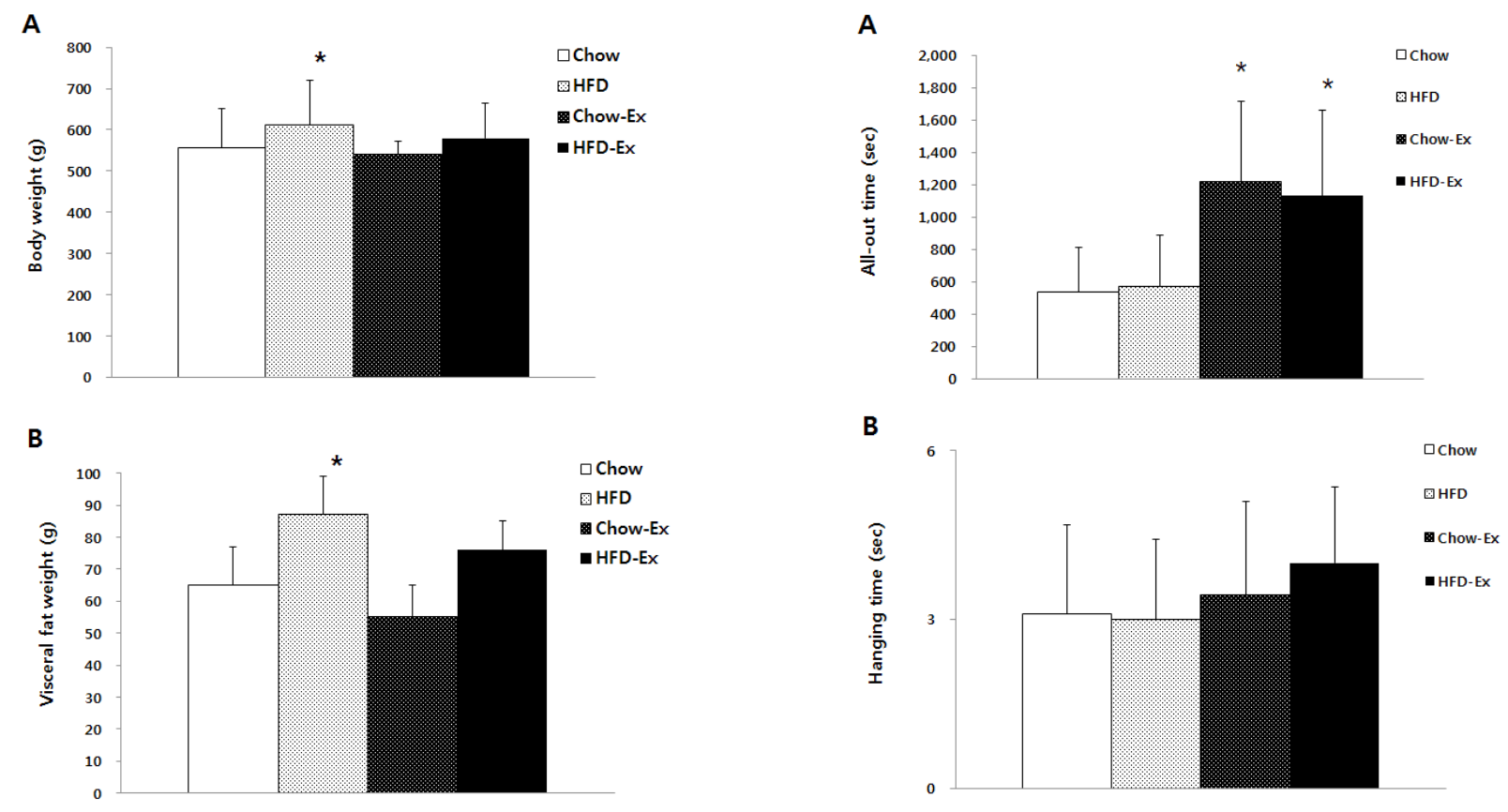

Figure 1. Comparisons of body weight (A) and visceral fat weight (B) after treatments $* p<0.05$ significant difference as compared to chow group.

Figure 2. Comparisons of endurance capacity (A) and muscular endurance (B) after treatments ${ }^{*} \mathrm{p}<0.05$ significant difference as compared to Chow and HFD groups.
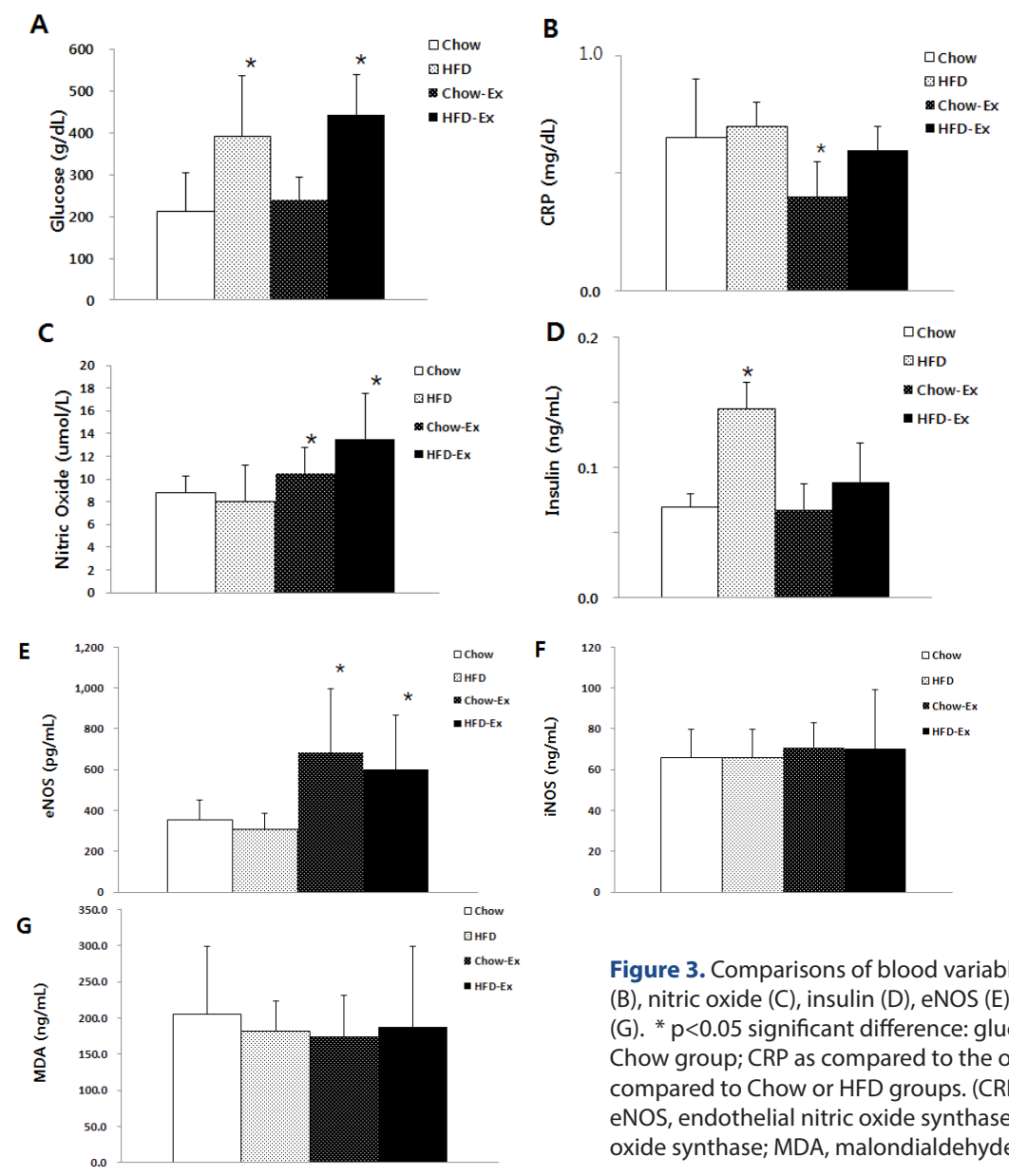

Figure 3. Comparisons of blood variables as glucose (A), CRP $(B)$, nitric oxide (C), insulin (D), eNOS (E), iNOS (F), and MDA (G). ${ }^{*} p<0.05$ significant difference: glucose as compared to Chow group; CRP as compared to the other groups; eNOS as compared to Chow or HFD groups. (CRP, C-reactive protein; eNOS, endothelial nitric oxide synthase; iNOS, inducible nitric oxide synthase; MDA, malondialdehyde). 


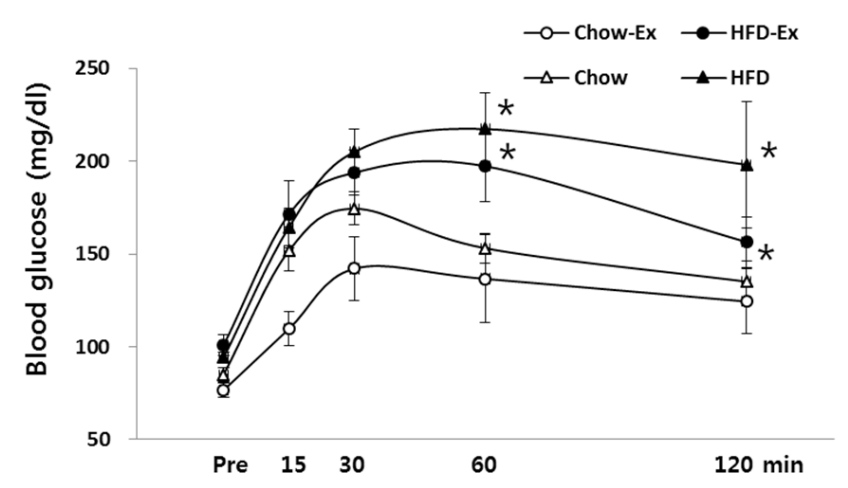

Figure 4. Comparisons of blood glucose concentration in OGTT after treatments * $p<0.05$ significant difference as compared to Chow or Chow-Ex group.

higher than the HFD and Chow groups who did not exercise, but there was no statistically significant difference.

The measurement results of blood variables are shown in Fig. 3, blood glucose concentration was significantly higher ( $\mathrm{p}$ $<0.05$ ) in both non-exercise HFD and exercise HFD groups than Chow group. Serum CRP levels were significantly lower $(\mathrm{p}<0.05)$ in the exercised Chow group than all the other groups. The blood NO level was significantly higher $(\mathrm{p}<0.05)$ in the HFD and Chow groups than in the non-exercise group. Blood insulin levels were significantly higher $(\mathrm{p}<0.05)$ in nonexercise HFD group than in all other groups. Blood eNOS activity was significantly higher $(\mathrm{p}<0.05)$ in the HFD and Chow groups than in the non-exercise group. Serum iNOS activity and MDA concentrations were not significantly different among groups.

In OGTT, the changes of in blood glucose concentrations were shown in Fig. 4. At 60 and 120 minutes of recovery, the non-exercise HFD and exercise HFD groups were significantly higher $(\mathrm{p}<0.05)$ than the Chow group. In the comparison based on the same diet, the exercise group was lower than the non-exercise group, but there was no statistically significant difference.

Comparison of protein expression in aortic tissue was shown in Fig. 5, IL-6 protein expression level was significantly higher $(\mathrm{p}<0.05)$ in the non-exercise HFD group than the other groups. The expression level of TNF- $\alpha$ was significantly lower $(\mathrm{p}<0.05)$ in the HFD and Chow groups than in the non-exercise group.
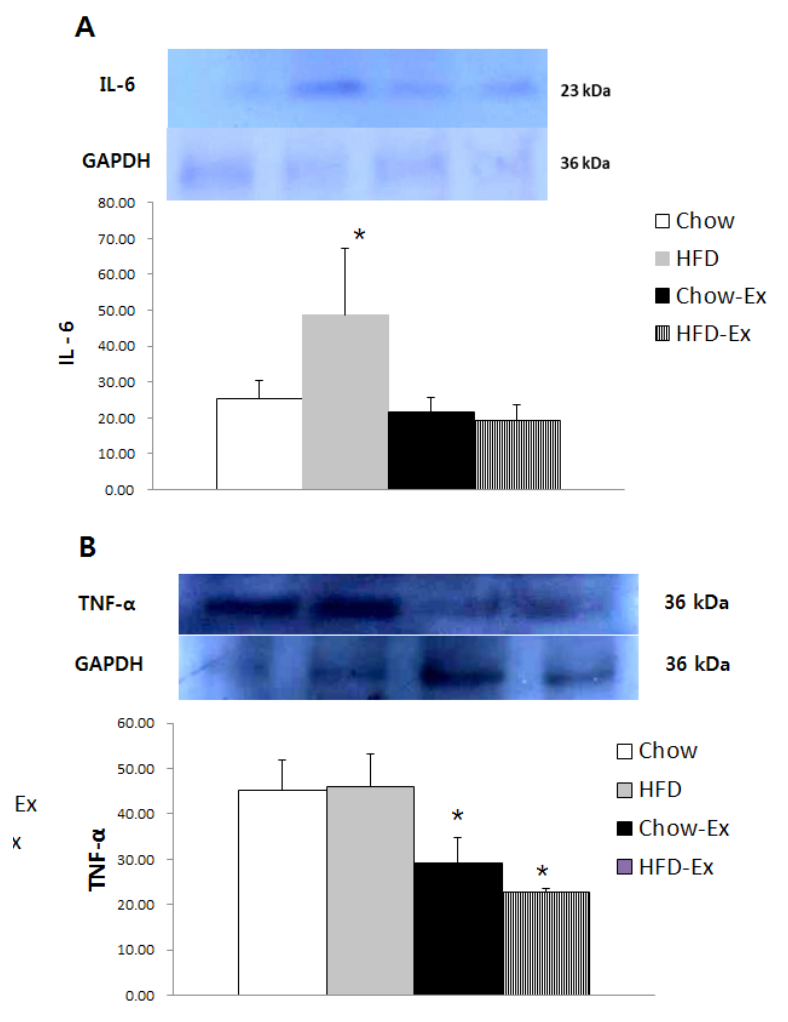

Figure 5. Comparisons of IL-6 and TNF-a protein expressions in aortic vascular tissue ${ }^{*} \mathrm{p}<0.05$ significant difference: IL- 6 as compared to the other groups; TNF- $a$ as compared to the Chow or HFD groups (IL-6, interleukin 6; TNF-a tumor necorsis factor alpha).

\section{Discussion}

Changes in body weight and visceral fat in this study showed a significant increase in the high-fat diet group, indicating the importance of dietary composition [17], which is regarded as an effective intervention of controlling body composition. However, significant body weight and visceral fat loss due to exercise performance could not be confirmed. Low-calorie and low fat intake is important as a basic method for reducing body weight and visceral fat mass [18], and in the case of exercise performance, an appropriate protocol based on more sufficient exercise amount is important. In other words, the exercise program for weight control confirms the need to be configured in more detail. In the change of physical fitness, endurance exercise ability was significantly improved in the exercise group, and there was no statistically significant difference in muscular endurance, but the exercise group also showed high exercise effect. It is considered that the exercise program applied in this study is a cardiovascular 
endurance improved as a treadmill running corresponding to aerobic exercise, and it was found that the exercise program had a slight effect on the improvement of muscle endurance of the long running. It is considered to reflect the specificity of exercise training [19].

The blood glucose level was higher in the high-fat diet group regardless of exercise, and the blood insulin level was higher in the high-fat diet group without exercise than in the Chow group. The exercise effect was confirmed by not showing this significant difference. Increased blood glucose and insulin concentrations in the high-fat diet group are considered to be a results of increased insulin resistance due to obesity [20]. These results are also clearly shown in the results of OGTT, the blood glucose concentration of OGTT showed a significant increase in the group fed high fat diet. In the case of exercise, low blood glucose levels were found in both types of diets, which suggests that exercise performance can alleviate the increase in insulin resistance caused by high-fat diet. The exercise effect related to improving insulin resistance improves the regulation of glucose metabolism by weight control, but the specific weight control effect by exercise training suppresses unnecessary gluconeogenesis and improves intracellular glucose homeostasis. This is due to the improvement of mitochondrial oxidation capacity [21]. In this study, however, the importance of dietary control was emphasized to improve insulin resistance, and it was considered important to construct more detailed exercise program to expect exercise effect.

Blood CRP levels have been considered to be important factors in inflammation and insulin resistance [22], and the Chow group with exercise was significantly lower than the non-exercise Chow group, confirming the effects of exercise to alleviate inflammation and insulin resistance. However, the HFD group did not show any significant difference even after exercise, so it was also possible to confirm the importance of dietary composition to relieve inflammation. Obesity and high-fat diets induce an inflammatory response with the accumulation of adipose tissue in the body, which acts as a cause of insulin resistance with an increase in inflammatory cytokine secretion. In this study, the high-fat diet group showed consistent results such as increased visceral fat, increased insulin resistance, and increased inflammatory factors.

Obesity due to high-fat diets is associated with a significant increase in risk factors for atherosclerosis, inhibition of nitric oxides that relax vascular endothelial cells, increased ET-1, which constricts vascular endothelial, and enhanced prostanoid-mediated vasoconstriction, and inflammatory activation [23]. In addition, it causes oxidative stress, increases secretion of inflammatory cytokines, inflammation of the vascular endothelium due to vascular wall deposition of platelets and monocytes, and increases blood CRP levels and atherosclerosis through the domino process of the decrease of nitric oxide secretion. In this study, it was confirmed that the high-fat diet intake group had a negative effect on vascular endothelial function by increasing blood CRP concentration and decreasing NO concentration. In chronic vascular diseases including atherosclerosis, the vascular endothelial layer exhibits decreased function such as vascular tension regulation, blood cell deposition, smooth muscle proliferation, and inflammatory response [24]. Nitric oxide in the vascular endothelial layer is the most representative factor for vascular relaxation function [25], which is a key factor in body composition, energy metabolism and vascular function regulation [26]. In particular, NO produced by eNOS in relation to vascular function plays a key role in maintaining homeostasis of blood vessel walls by inhibiting platelet deposition, inflammation, oxidative stress, vascular smooth muscle cell migration and proliferation, and leukocyte deposition [27], also plays an important role in preventing inflammation of blood vessel tissue [28]. NO concentration and eNOS activity are known to be inhibited in obesityinduced obesity by high-fat diets [29], whereas iNOS is known to affect inflammatory macrophages [30]. In this study, blood NO concentration and eNOS activity were higher in the exercise group in both the high fat diet and the Chow diet, indicating that the positive effect of exercise performance was higher than the diet composition. The effect of shear stress on blood vessels has an important effect on blood vessel elasticity control [31]. When blood flow and shear stress increase through exercise, eNOS is activated and nitric oxide secretion increases. It has a positive effect on the activation 
of endothelial function and cardiovascular function [32]. This effect of exercise not only activates vascular endothelial function in healthy adults, obese, postmenopausal women, the elderly, and hyperlipidemic patients, but also improves the vascular endothelial bioavailability in patients with coronary artery disease and heart failure, which is effective in treating these diseases [33, 34]. In particular, aerobic exercise has been reported to have a positive effect on the treatment of cardiovascular disease through increased secretion of nitric oxide and activation of bioavailability [35]. In this study, the high-fat diet group showed a significant decrease in blood NO and eNOS activity, and the exercise group showed a significant increase in blood NO and eNOS activity. In contrast, it was clearly confirmed that aerobic exercise had a positive effect.

As a result of analysis of protein expression level of aortic vascular tissue, HFD group was significantly higher in IL-6 protein expression, and high-fat diet was considered to have a negative effect on inflammation of vascular tissues. In the Chow group and the high-fat diet group, exercise significantly decreased, indicating that exercise performance had a positive effect on the inflammation of vascular tissues. In this study, the protein expression of Il- 6 and TNF- $\alpha$ [36], which are representative factors of obesity-related inflammatory cytokines, were significantly increased in the vascular tissues of the high-fat diet group. It was confirmed that it affects the increase in insulin resistance, and as a result, it was confirmed that obesity is likely to indicate the activation of vascular inflammation [37]. TNF- $\alpha$ is one of the most likely inflammation-inducing cytokines during obesity, and NO bioavailability and the inhibition of eNOS protein expression in vascular tissues are inextricably linked with TNF- $\alpha$. TNF- $\alpha$ acts as a key cause of endothelial dysfunction in which ROS is increased and impairs NO-mediated endothelium-dependent vasodilation $[1,38,39]$. In the obese state, it is highly likely that the regulation of vascular function is difficult due to the secretion of substances that negatively affect vascular function from perivascular adipose tissue (PVAT) [40]. Sousa et al. [41] suggest that PVAT is an important cause of endothelial dysfunction by releasing inflammatory markers and oxidative stressors in the blood, and aerobic exercise is thought to be an up-regulated the anti-oxidant expression and decreased
PVAT oxidative stress. It helps to improve endothelial relaxation. Therefore, it can be seen that aerobic exercise of this study may help to improve vascular endothelial function that can be decreased after obesity caused by high fat diet. In this study, variables that directly affect NO availability, such as NOS coupling or folic acid concentrations [42], were not measured, but it is clearly confirmed that exercise helped to improve vascular endothelial function by increasing blood $\mathrm{NO}$ and eNOS activity. In addition, as the exercise groups with chow or high-fat diet significantly reduced the expression of TNF- $\alpha$ protein as inflammatory marker [43] in blood vessels and improved blood NO concentration and eNOS activity, it has been clearly shown that exercise can relieve vascular inflammation and have a significant impact on vascular endothelial activation.

In addition, Brunn et al. [44] also reported a significant decrease in inflammation-related markers such as CRP, IL-6, IL-8 and MCP-1 in the blood through exercise and low calorie diet. Therefore, exercise performance and dietary changes may be very effective considerations for improving vascular endothelial function and inflammation.

\section{Conclusion}

High-fat dietary intake leads to an increase in body weight, visceral fat mass, insulin resistance and inflammatory factors, and is likely to cause a decrease in vascular endothelial function. The results of this study confirmed that exercise training through aerobic exercise and dietary control may help to reduce inflammatory expression and improve vascular endothelial function in high-fat diet induced obese rats.

\section{Acknowledgments}

This work was supported by the Ministry of Education of the Republic of Korea and the National Research Foundation of Korea (NRF-2018S1A5A2A01039065). This paper is an amendment and supplement to what was oral presented at the 10th Asia Conference on Kinesiology on August 8-11 of 2019 at UESTC. 


\section{Conflicts of Interest}

The author declared no conflicts of interest.

\section{References}

1. Lind L, Carlsson AC, Siegbahn A, Sundström J, Anlöv J. Impact of physical activity on cardiovascular status in obesity. Eur J Clin Invest. 2017; 47(2):167-175.

2. Vinet A, Karpoff L, Walther G, et al. Vascular reactivity at rest and during exercise in middle-aged obese men: effects of short-term, low-intensity, exercise training. Int J Obesity. 2010; 35(6):820-828.

3. Addison O, LaStayo PC, Dibble LE, Marcus RL. Inflammation, aging, and adiposity: implications for physical therapists. J Geriat Phys Ther. 2012; 35(2):8694.

4. Virdis A. Endothelial dysfunction in obesity: role of inflammation. High Blood Press Cardiovasc Prev. 2016; 23(2):83-85.

5. Tsalamandris S, Antonopoulos AS, Oikonomou E, et al. The role of inflammation in diabetes: current concepts and future perspectives. Eur Cardiol. 2019; 14(1):50-59.

6. Noslaski R, Guzik TJ. Perivascular adipose tissue inflammation in vascular disease. British J Pharmacol. 2017; 174:3496-3513.

7. Zou L, Wang W, Liu S, et al. Spontaneous hypertension occurs with adipose tissue dysfunction in perilipin-1 null mice. Biochim Biophys Acta. 2016; 1862:182-191.

8. Taddei S, Galetta F, Virdis A. Ghiadoni L, et al. Physical activity prevents age-related impairment in nitric oxide availability in elderly athletes. Circulation. 2000; 101(25):2896-2901.

9. Franzoni F, Ghiadoni L, Galetta F, et al. Physical activity, plasma antioxidant capacity, and endotheliumdependent vasodilation in young and older men. Am J Hyperten. 2005; 18(4):510-516.

10. Beavers KM, Hsu FC, Isom S, et al. Long-term physical activity and inflammatory biomarkers in older adults. Med Sci Sports \& Exerc. 2010; 42(12):2189-2196.

11. Carlsson AC, Arnlov J, Sundstrom J, Michaelsson K,
Byberg L, Lind L. Physical activity, obesity and risk of cardiovascular disease in middle-aged men during a median of 30 years of follow-up. Eur J Prevent Cardiol. 2015; 23(4):359-365.

12. Pedrinolla A, Venturelli M, Kirmizi E, et al. Role of exercise in vascullar function and inflammatory profile in age-related obesity. J Immunol Res. 2018; Article ID 7134235 .

13. Ivy JL, Young JC, McLane JA, Fell RD, Holloszy JO. Exercise training and glucose uptake by skeletal muscle in rats. J Appl Physiol Respir Environ Exerc Physiol. 1983; 55:1393-1396.

14. Simi B, Sempore B, Mayet MH, Favier RJ. Additive effects of training and high-fat diet on energy metabolism during exercise. J Appl Physiol. 1991; 71(1):197-203.

15. Park JS, Kim JS, Lee MG, Treadmill exercise effect to the macrophage transformation markers, electromyography (EMG), grip strength and holding ability after the peripheral nerve injury. Kor J Spt Sci. 2015; 24(6):10011011

16. Lowry EC, Blumber JM, Rhea RL, Ranson JP. Serum levels of orally administered penicillin. US Armed Forces Med J. 1951; 2(2):265-270.

17. Hamdy O, Tasabehji MW, Elseaidy T, Tomah S, Ashrafzadeh S, Mottalib A. Fat versus carbohydratebased energy-restricted diets for weight loss in patients with type 2 diabetes. Curr Diab Rep. 2018; 18(12):128.

18. Shai I, Schwarzfuchs D, Henkin Y, et al. Weight loss with a low-carbohydrate, Mediterranean, or low-fat diet. N Engl J Med. 2008; 359:229-241.

19. Romero SA, Minson CT, Halliwill JR. The cardiovascular system after exercise. J Appl Physiol. 2017; 122(4):925932.

20. Choi CS. Pathogenesis of insulin resistance. Kor J Internal Med. 2009; 77(2):171-177.

21. Keshel TE, Coker RH. Exercise training and insulin resistance: a current review. J Obes Weight Loss Ther. 2015; 5 .

22. Brunner EJ, Kivimäki M, Witte DR, et al. Inflammation, insulin resistance, and diabetes--Mendelian randomization using CRP haplotypes points upstream. 
PLoS Med. 2008;12; 5(8):e155.

23. Cardillo C, Campia U, Iantorno M, Panza JA. Enhanced vascular activity of endogenous endothelin-1 in obese hypertensive patients. Hypertension. 2004; 43:36-40.

24. Park K, Park WJ. Endothelial dysfunction: clinical implications in cardiovascular disease and therapeutic approaches. J Korean Med Sci. 2015; 30:1213-1225.

25. Mudau M, Genis A, Lochner A, Strijdom H. Endothelial dysfunction: the early predictor of atherosclerosis. Cardiovasc J Afr. 2012; 23:222-231.

26. Atawia RT, Bunch KL, Toque HA, Caldwell RB, Caldwell RW. Mechanisms of obesity-induced metabolic and vascular dysfunctions. Front Biosci(Landmark Ed). 2019; 24:890-934.

27. Tousoulis D, Simopoulou C, Papageorgiou N, et al. Endothelial dysfunction in conduit arteries and in microcirculation. Novel therapeutic approaches. Pharmacol Ther. 2014; 144:253-267.

28. Li H, Xia N, Förstermann U. Nitric Oxide Synthesis in Vascular Physiology and Pathophysiology In: Endothelial Signaling in Development and Disease. Springer, 2015.

29. Sansbury BE, Cummins TD, Tang Y, et al. Overexpression of endothelial nitric oxide synthase prevents dietinduced obesity and regulates adipocyte phenotype. Circulation Research. 2012; 111(9):1176-1189.

30. Jang JE, Ko MS, Yun J-Y, et al. Nitric oxide produced by macrophages inhibits adipocyte differentiation and promotes profibrogenic responses in preadipocytes to induce adipose tissue fibrosis. Diabetes. 2016; 65(9):2516-2528.

31. Andreou I, Antoniadis AP, Shishido K, et al. How do we prevent the vulnerable atherosclerotic plaque from rupturing? Insights from in vivo assessments of plaque, vascular remodeling, and local endothelial shear stress. J Cardiovasc Pharmacol Ther. 2015; 20:261-275.

32. Thijssen DH, Dawson EA, Black MA, Hopman MT, Cable NT, Green DJ. Brachial artery blood flow responses to different modalities of lower limb exercise. Med Sci Sports Exerc. 2009; 41:1072-1079.

33. Barton M. Prevention and endothelial therapy of coronary artery disease. Curr Opin Pharmacol. 2013; 13:226-241.

34. Wienbergen $H$, Hambrecht R. Physical exercise and its effects on coronary artery disease. Curr Opin Pharmacol. 2013; 13:218-225.

35. da Silva Sponton AC, Sousa AS, Delbin MA. Vascular dysfunction in obesity: Beneficial effects of aerobic exercise training in animal models. Motriz Rio Claro. 2017; 23:e101621.

36. Exley MA, Hand L, O'Shea D and Lynch L. Interplay between the immune system and adipose tissue in obesity. J Endocrinol. 2014; 223(2):R41-R48.

37. Airaksinen K, Jokkala J, Ahonen I, et al. High-fat diet, betaine, and polydextrose induce changes in adipose tissue inflammation and metabolism in C57BL/6J mice. Mol Nutr Food Res. 2018; 62(23):e1800455.

38. Galili O, Versari D, Sattler KJ, et al. Early experimental obesity is associated with coronary endothelial dysfunction and oxidative stress. Am J Physiol Heart Circ Physiol. 2007; 292(2):H904-H911.

39. Kleinbongard P, Heusch G, Schulz R. TNFalpha in atherosclerosis, myocardial ischemia/reperfusion and heart failure. Pharmacol Ther. 2010; 127:295-314.

40. Boa BCS, Yudkin JS, van Hinsbergh VWM, Bouskela E, Eringa EC. Exercise effects on perivascular adipose tissue: endocrine and paracrine determinants of vascular function. British J Pharmacol. 2017; 174(20):3466-3481.

41. Sousa AS, Sponton ACS, Trifone CB, Delbin MA. Aerobic exercise training prevents perivascular adipose tissueinduced endothelial dysfunction in thoracic aorta of obese mice. Frontiers in Physiology. 2019; 10.

42. Chen J, Te Z, Wang X, et al. Nitric oxide bioavailability dysfunction involves in atherosclerosis. Biomed Pharmacotherapy. 2018; 97:423-428.

43. Zhang H, Park Y, Wu J, et al. Role of TNF- $\alpha$ in vascular dysfunction. Clin Sci (Lond). 2009; 116:219-230.

44. Bruun JM, Helge JW, Richelsen B, Stallknecht B. Diet and exercise reduce low-grade inflammation and macrophage infiltration in adipose tissue but not in skeletal muscle in severely obese subjects. Am J PhysiolEndocrinol Metabolism. 2006; 290(5):E961-E967. 